OCTOBER, 1974

\title{
GENERALIZED PLANE DEFORMATIONS OF IDEAL FIBER-REINFORCED MATERIALS*
}

BY

\author{
A. C. PIPKIN \\ Brown University
}

1. Introduction. Finite deformations of materials composed of long, strong fibers bonded together with a weaker matrix material can be studied by using the theory of ideal fiber-reinforeed materials. This theory, which is the subject of a recent book by Spencer [1], is based on the idealizations that the fibers are continuously distributed and inextensible and that the composite is incompressible in bulk. In plane deformations of such materials [2], the kinematic constraint conditions are sufficiently restrictive, so that stress-strain relations often play only a minor role. For this reason, it is relatively easy to solve problems that would be intractible if the material were isotropic [3-10]. The relation of solutions from the idealized theory to solutions for slightly extensible materials is understood fairly well $[11,12]$. Most of the existing work on plane deformations is discussed in a recent review article [13].

In the present paper we discuss deformations of cylindrical bodies with fibers lying in cross-sectional planes. The fibers may be curved, but they have the same distribution on each cross-section. The body is stretched or compressed in the axial direction and then subjected to a further plane deformation. The axial stretching causes the cross-section to change shape. The theory of superposed plane deformations is not essentially different from the theory that applies when there is no stretching [2], if the stretched state is used as the reference state. Consequently, our main object is to determine the state of deformation produced by axial stretching.

Because of the cross-sectional distortion produced by stretching in the axial direction, it may or may not be possible to accomplish this stretching by means of a simple tensile stress alone, depending on the arrangement of fibers on each cross-section. For some arrangements, axial stretching necessarily requires shearing parallel to the fiber direction. With any reasonable assumption about the stress response, this implies that the stress in the stretched state is not merely an axial tension.

We approach the problem of determining the cross-sectional distortion by an inverse method in which it is not necessary to specify the stress response in advance. We determine the fiber arrangements that will allow axial stretching with no shearing parallel to the fibers. We show that the kinematical condition of no shearing can be satisfied only if the fibers and their orthogonal trajectories form a Hencky-Prandtl net when the body is undeformed (Sec. 6).

\footnotetext{
* Received March 13, 1973. The work described in this paper was carried out under grant GP-28881
} from the National Science Foundation to Brown University. 
Fortunately, these cases include all that are likely to be of any practical importance. When the fibers are initially straight and parallel, or when they lie on concentric circles, or in any case in which the fibers lie along parallel curves, the fibers and their orthogonal trajectories do form a Hencky-Prandtl net. The deformation produced by axial stretching for all cases with initially parallel fibers is determined in Sec. 7, and for cases involving straight but non-parallel fibers in Sec. 8. The general Hencky-Prandtl net is discussed in Sec. 9.

Because our main results are purely kinematical, discussion of stress response is deferred until Sec. 10. We consider only elastic stress response, as an illustration. The stress equilibrium equations are not used until Sec. 11. We use them to show that if the shearing stress is zero whenever there is no shearing, then stretching with no shearing can indeed be supported by axial tension alone. In Sec. 12 we briefly verify that problems of superposed plane deformation do reduce to ordinary plane problems if the stretched state is used as the reference state.

2. Fibers and normal lines. We consider a cylindrical body of arbitrary, uniform cross-section, with its axis in the $Z$-direction of a rectangular Cartesian coordinate system $X, Y, Z$. We suppose that the body is stretched (or compressed) to $\lambda$ times its initial length in the axial direction and subjected to a plane deformation as well. The particle initially at $\mathbf{X}$ moves to a place $\mathbf{X}(\mathbf{X})$ given in Cartesian coordinates by a mapping of the form

$$
x=x(X, Y), \quad y=y(X, Y), \quad z=\lambda Z .
$$

The body is reinforced with inextensible fibers, which are treated as continuously distributed. Through each point of the body there is a material curve that we call a fiber. The fibers lie in cross-sectional planes and have the same arrangement on each crosssection. In the undeformed body this arrangement is specified by giving the angle $\theta_{0}(X, Y)$ between the fiber direction at the point $\mathbf{X}$ and some fixed direction. After the deformation, the fibers on a given cross-section form a new family of plane curves, and the fiber angle at $\mathbf{x}$ is denoted $\theta(x, y)$.

The orthogonal trajectories of the fibers on a given cross-section are called normal lines. Let $\mathbf{a}$ and $\mathbf{n}$ be unit vectors tangential to the fiber and the normal line at $\mathbf{x}$. These vectors can be expressed as functions of the fiber angle $\theta$ at $\mathbf{x}$. If we take the $x$-direction to be the direction $\theta=0$ (which is not always convenient), then in terms of constant base vectors $\mathbf{i}, \mathbf{j}, \mathbf{k}$ in the coordinate directions, we have

$$
\mathrm{a}(\theta)=\mathbf{i} \cos \theta+\mathbf{j} \sin \theta
$$

and

$$
\mathbf{n}(\theta)=-\mathbf{i} \sin \theta+\mathbf{j} \cos \theta .
$$

The same functions of $\theta$, evaluated at $\theta_{0}(X, Y)$, give unit vectors along the fibers and normal lines in the undeformed body. For abbreviation, we denote these by $\mathbf{a}_{0}$ and $\mathbf{n}_{0}$. We note that the derivatives of $\mathbf{a}$ and $\mathbf{n}$ with respect to $\theta$ are

$$
\mathbf{a}^{\prime}(\theta)=\mathbf{n}(\theta), \quad \mathbf{n}^{\prime}(\theta)=-\mathbf{a}(\theta) .
$$

The gradient with respect to initial position $\mathbf{X}$ is denoted by $\nabla_{0}$ and the gradient with respect to $\mathrm{x}$ is $\nabla$. The radii of curvature of fibers and normal lines after the deforma- 
tion, $r_{a}$ and $r_{n}$, are defined by

$$
1 / r_{a}=\mathbf{a} \cdot \nabla \theta, \quad 1 / r_{n}=\mathbf{n} \cdot \nabla \theta .
$$

Similarly, the initial radii of curvature are given by

$$
1 / r_{a 0}=\mathbf{a}_{0} \cdot \nabla_{0} \theta_{0}, \quad 1 / r_{n 0}=\mathbf{n}_{0} \cdot \nabla_{0} \theta_{0} .
$$

Directional derivatives in the fiber and normal directions generally do not commute. Instead, we find that

$$
(\mathbf{n} \cdot \boldsymbol{\nabla})(\mathbf{a} \cdot \boldsymbol{\nabla})-(\mathbf{a} \cdot \boldsymbol{\nabla})(\mathbf{n} \cdot \boldsymbol{\nabla})=[(\mathbf{n} \cdot \boldsymbol{\nabla}) \mathbf{a}-(\mathbf{a} \cdot \boldsymbol{\nabla}) \mathbf{n}] \cdot \boldsymbol{\nabla} .
$$

By using (2.4) and (2.5) to simplify the right-hand member, we obtain

$$
(\mathbf{n} \cdot \boldsymbol{\nabla})(\mathbf{a} \cdot \boldsymbol{\nabla})-(\mathbf{a} \cdot \boldsymbol{\nabla})(\mathbf{n} \cdot \boldsymbol{\nabla})=\left(\mathbf{n} / r_{n}+\mathbf{a} / r_{a}\right) \cdot \nabla .
$$

The initial fields satisfy the same commutation relation.

3. Constraints. Material line elements of length $d s$ in the fiber, normal, and axial directions before the deformation are carried by the deformation $\mathbf{x}(\mathbf{X})$ onto elements with lengths and directions defined by

$$
\left(\mathbf{a}_{0} \cdot \nabla_{0}\right) \mathbf{x}(\mathbf{X}) d s, \quad\left(\mathbf{n}_{0} \cdot \nabla_{0}\right) \mathbf{x}(\mathbf{X}) d s, \quad\left(\mathbf{k}_{0} \cdot \nabla_{0}\right) \mathbf{x}(\mathbf{X}) d s,
$$

respectively. These directions and lengths are restricted by certain kinematical constraints imposed on admissible deformations.

Because we consider generalized plane deformations, the initially axial element is still in the axial direction and of length $\lambda d s$ after the deformation. Hence,

$$
\left(\mathbf{k}_{0} \cdot \nabla_{0}\right) \mathbf{x}(\mathbf{X})=\lambda \mathbf{k}
$$

The element initially in the fiber direction $\mathbf{a}_{0}$ still lies along a fiber after the deformation, and is thus in the direction a. Because we treat fibers as inextensible, its length is still $d s$. Thus,

$$
\left(\mathrm{a}_{0} \cdot \nabla_{0}\right) \mathbf{x}(\mathrm{X})=\mathbf{a} .
$$

The element initially in the normal direction does not necessarily lie in the normal direction after the deformation, but it is in the plane of a and $\mathbf{n}$. Its component in the $\mathbf{n}$-direction is determined by a further constraint. We treat the composite as incompressible in bulk, so the parallelepiped spanned by the elements (3.1) must have the volume $(d s)^{3}$. This implies that the element initially in the normal direction has the component $d s / \lambda$ in the $\mathbf{n}$-direction after the deformation:

$$
\left(\mathbf{n}_{0} \cdot \nabla_{0}\right) \mathbf{x}(\mathbf{X})=\lambda^{-1}(\mathbf{n}+\kappa \mathbf{a}) .
$$

The coefficient $\kappa$ represents the amount of shear parallel to the fiber direction, measured with respect to the stretched state.

Since there is a one-to-one correspondence between initial position $\mathbf{X}$ and final position $\mathbf{x}$, we can treat quantities associated with a given particle as functions of either $\mathbf{x}$ or $\mathbf{X}$, as convenience dictates. By using (3.2) to (3.4) and the chain rule, we find that derivatives with respect to the two sets of variables are related by

$$
\mathbf{a}_{0} \cdot \nabla_{0}=\mathbf{a} \cdot \boldsymbol{\nabla}, \quad \mathbf{n}_{0} \cdot \nabla_{0}=\lambda^{-1}(\mathbf{n}+\kappa \mathbf{a}) \cdot \nabla, \quad \mathbf{k}_{0} \cdot \nabla_{0}=\lambda \mathbf{k} \cdot \nabla .
$$


4. Compatibility conditions. The constraints (3.2)-(3.4) restrict the deformations $\mathbf{x}(\mathbf{X})$ that can be considered. Because of these constraints, the functions $\theta(x, y)$ and $\kappa(x, y)$ that specify the fiber angle and amount of shear cannot be specified arbitrarily or independently. To obtain compatibility conditions on these variables, i.e., integrability conditions for the system of equations (3.2) to (3.4), we eliminate $\mathbf{x}(\mathbf{X})$ from (3.3) and (3.4) by cross-differentiation. In doing this, we use the commutator (2.8), written for the initial fields. We obtain

$$
\left(\mathbf{n}_{0} \cdot \nabla_{0}\right) \mathbf{a}-\left(\mathbf{a}_{0} \cdot \nabla_{0}\right) \lambda^{-1}(\mathbf{n}+\kappa \mathbf{a})=\lambda^{-1}(\mathbf{n}+\kappa \mathbf{a}) / r_{n 0}+\mathbf{a} / r_{a 0} .
$$

By using (2.4) here and then separating the resulting equation into components in the fiber and normal directions, we obtain

$$
\mathbf{a}_{0} \cdot \nabla_{0}(\theta-\kappa)=\lambda / r_{a 0}+\kappa / r_{n 0}
$$

and

$$
\lambda \mathbf{n}_{0} \cdot \nabla_{0} \theta-\kappa \mathbf{a}_{0} \cdot \nabla_{0} \theta=1 / r_{n 0} .
$$

By using (3.5) and (2.5), we can rewrite (4.3) as

$$
\mathbf{n} \cdot \nabla \theta=\mathbf{n}_{0} \cdot \nabla_{0} \theta_{0}, \quad \text { or } r_{n}=r_{n 0} .
$$

Thus, just as in ordinary plane deformations [2], the radius of curvature of the normal line through a particle after the deformation is the same as the radius of curvature of the normal line that passed through it before the deformation.

We can also rewrite (4.2) as either

$$
\mathbf{a}_{0} \cdot \nabla_{0}\left(\theta-\lambda \theta_{0}-\kappa\right)=\kappa \mathbf{n}_{0} \cdot \nabla_{0} \theta_{0}
$$

or

$$
\mathbf{a} \cdot \nabla\left(\theta-\lambda \theta_{0}-\kappa\right)=\kappa \mathbf{n} \cdot \nabla \theta .
$$

In the particularly important cases in which normal lines are initially straight (and thus straight in any state of deformation), the right-hand member of (4.6) is zero and the equation can be integrated immediately. In these cases the amount of shear $\kappa$ is equal to $\theta-\lambda \theta_{0}$ plus an amount that is constant on cach fiber.

5. Simple axial tension. In infinitesimal deformations, with linear equations, generalized plane strain amounts to the sum of two solutions, axial stretching and a plane deformation. For the finite deformations that we consider here, solutions cannot be superposed additively, but we can still break the solution into two parts: first the axial stretching and then, with the stretched state as reference state, an ordinary plane deformation. Since problems of plane deformation have been discussed elsewhere, we concentrate here on the first part of the problem, the determination of the state of deformation produced by uniform stretching in the axial direction with no tractions on the lateral surfaces of the body.

This problem, which is trivial for isotropic materials, is not entirely simple in the present context. The constraint conditions require the cross-section to change shape when the body is stretched in the axial direction, and for an arbitrary initial field of fiber directions $\theta_{0}(X, Y)$, determination of the cross-sectional shape after axial stretching is no simpler than any other plane problem. 
We do not consider even the stretching problem in all generality, but restrict attention to cases in which the axial stretching can be accomplished with only a tensile stress $\sigma_{z z}$. Although we defer discussion of stress response until Sec. 10, it is probably obvious that under loose assumptions about the stress, the stress components $\sigma_{x x}, \sigma_{y y}$, and $\sigma_{x y}$ can all be zero only if the amount of shear $\kappa$ is zero everywhere. Thus, although we speak of simple axial tension, our problem is a purely kinematical one. We wish to determine whether or not there is a state of deformation with $\kappa=0$ everywhere, and if so, what it is.

6. Fiber arrangements allowing simple axial tension. We now show that a state of simple axial tension is possible only if the fibers and normal lines initially form a HenckyPrandtl net, and that if they do, they again form another Hencky-Prandtl net after the axial stretching.

In deformations with no shearing, i.e. $\kappa=0$ everywhere, normal lines behave as material lines. Conversely, deformations in which all normal lines map onto normal lines have zero shear everywhere. A line element $\mathbf{n}_{0} d s$ maps onto $\mathbf{n} d s / \lambda$ in the deformation, and thus remains in the normal direction, as we see from (3.4) with $\kappa=0$.

In such cases (3.5) takes the simpler form

$$
\mathbf{a}_{0} \cdot \nabla_{0}=\mathbf{a} \cdot \nabla, \quad \lambda \mathbf{n}_{0} \cdot \nabla_{0}=\mathbf{n} \cdot \nabla, \quad \mathbf{k}_{0} \cdot \nabla_{0}=\lambda \mathbf{k} \cdot \nabla,
$$

and the compatibility conditions (4.4) and (4.6) then become

$$
\mathbf{a} \cdot \boldsymbol{\nabla}\left(\theta-\lambda \theta_{0}\right)=0, \quad \mathbf{n} \cdot \boldsymbol{\nabla}\left(\lambda \theta-\theta_{0}\right)=0 .
$$

Equivalently,

$$
r_{a}=r_{a 0} / \lambda, \quad r_{n}=r_{n 0} .
$$

Let $\xi$ be a coordinate constant along normal lines and let $\eta$ be constant along fibers. Then from (6.2) we obtain

$$
\theta-\lambda \theta_{0}=f(\eta), \quad \lambda \theta-\theta_{0}=g(\xi) .
$$

Let $A B C D$ be a curvilinear region bounded by fibers along $A B$ and $C D$ and by normal lines along $A C$ and $B D$. Then, with a little algebra, we find from (6.4) that the angles $\theta$ at the four corners satisfy the relation

$$
\theta_{A}-\theta_{B}=\theta_{C}-\theta_{D}
$$

and the same relation is satisfied by the initial angles $\theta_{0}$ at the four corner particles.

When two orthogonal families of curves have the property (6.5), they form a HenckyPrandtl net. The geometrical properties of such nets have been investigated thoroughly in connection with plane plastic deformations (see Prager and Hodge [14], for example). Thus, we have found that axial stretching can be carried out with no shearing anywhere only if the fibers and normal lines initially are arranged in a Hencky-Prandtl net.

Such an arrangement is sufficient to allow a deformation with no shearing, because with the compatibility conditions satisfied, the relations (3.2) to (3.4) can be integrated to yield a deformation $\mathbf{x}(\mathbf{X})$. In Secs. 7 and 8 we construct the deformation explicitly for the special cases in which either normal lines or fibers are initially straight. In Sec. 9 we consider the cases in which neither fibers nor normal lines are straight. 
7. Initially parallel fibers. Because of practical limitations on methods of fabrication, it will most usually be the case that the fibers lie along curves that are initially parallel, although not necessarily straight, such as concentric circles. In such cases the normal lines are initially straight, but not necessarily parallel. Almost all solved problems in the literature deal with such cases. They are especially simple because the normal lines must remain straight in every state of deformation, as remarked in Sec. 4.

It is trivial to verify that any net in which one family is composed of straight lines is a Hencky-Prandtl net. Thus, uniform axial stretching with no shearing is always kinematically admissible when the fibers are initially parallel. The simplest cases are those in which the fibers are not only parallel but straight. In these cases, if we take the $X$-direction parallel to the fibers, the deformation is

$$
x=X, \quad y=Y / \lambda, \quad z=\lambda Z .
$$

Both here and in the following examples, we leave aside an arbitrary rigid motion. In this case the distortion of the cross-section is a simple contraction perpendicular to the fibers, of an amount sufficient to preserve the volume.

In the more general cases in which fibers are parallel but curved, we select some straight normal line and designate the fiber angle on it as $\theta_{0}=0$. After the axial stretching deformation, the normal line is still straight, and with no loss of generality we can designate the fiber angle on it as $\theta=0$. Then $\theta-\lambda \theta_{0}$ is zero on that normal line, and also on all the fibers that cross it, according to $(6.2 \mathrm{a})$. Then, by a repetition of the argument if necessary, the same is true everywhere if the cross-section is connected:

$$
\theta=\lambda \theta_{0} .
$$

As a special case, suppose that fibers initially lie on concentric circles in the region $R_{1} \leq R \leq R_{2}, 0 \leq \theta_{0} \leq A$, where $R$ and $\theta_{0}$ are polar coordinates. There is no inconsistency in calling $\theta_{0}$ the fiber angle; we simply take the $Y$-direction as the fiber direction for which the fiber angle is zero. The polar coordinate $R$ is the same as the fiber radius of curvature, $r_{a 0}$. Then from (6.3a) and (7.2), we find that in simple axial tension the particle initially at $R, \theta_{0}, Z$ moves to $r, \theta, z$, where

$$
r=R / \lambda, \quad \theta=\lambda \theta_{0}, \quad z=\lambda Z \text {. }
$$

With $\lambda>1$, the curvilinear block becomes thinner in the radial direction and curls up more tightly. If $\lambda=2 \pi / A$, the two plane faces initially along $\theta_{0}=0$ and $\theta_{0}=A$ meet at $\theta=0(\theta=2 \pi)$, and a tube is formed.

For any case with initially parallel fibers, the body can be regarded as separated into infinitesimal wedges by the straight normal lines, and each wedge deforms as the finite wedge in the preceding example did. For an analytical representation of the deformation, we label each normal line with the fiber angle $\theta_{0}$ on it, and we label each fiber with its distance $\eta$ from some arbitrarily selected fiber. The net is completely defined when the radius of curvature $r_{a 0}$ along the fiber $\eta=0$ is specified as a function of $\theta_{0}$. If we call this $r_{0}\left(\theta_{0}\right)$, then because the fibers are parallel, $r_{a(0)}$ is given everywhere by

$$
r_{a 0}=r_{0}\left(\theta_{0}\right)-\eta \text {. }
$$

We specify the deformation by expressing both $\mathbf{X}$ and $\mathbf{x}$ in terms of the parameters $\theta_{0}, \eta$, and $Z$. Taking note of the expressions for elements of are length in the initial fiber, normal, and axial directions, we write 


$$
d \mathbf{X}=\mathbf{a}_{0} r_{a 0} d \theta_{0}+\mathbf{n}_{0} d \eta+\mathbf{k}_{0} d Z .
$$

By using (2.4) and (7.4) we find that the right-hand member of (7.5) is indeed a perfect differential, and we obtain

$$
\mathbf{X}=\int_{0}^{\theta_{0}} \mathbf{a}(\alpha) r_{0}(\alpha) d \alpha+\mathbf{n}\left(\theta_{0}\right) \eta+\mathbf{k}_{0} Z .
$$

To express the final position $\mathbf{x}$ in terms of the same parameters, we first combine (3.2) to (3.4) (with $\kappa=0$ ) as

$$
d \mathbf{x}=\mathbf{a} r_{a 0} d \theta_{0}+\lambda^{-1} \mathbf{n} d \eta+\lambda \mathbf{k} d Z .
$$

With a and $\mathrm{n}$ the functions of $\theta$ defined in (2.2) and (2.3), $\theta$ given in terms of $\theta_{0}$ by (7.2), and $r_{a 0}$ given by (7.4), then the right-hand member of (7.7) is a perfect differential, and integration gives

$$
\mathbf{x}=\int_{0}^{\theta_{0}} \mathbf{a}(\lambda \alpha) r_{0}(\alpha) d \alpha+\lambda^{-1} \mathbf{n}\left(\lambda \theta_{0}\right) \eta+\lambda \mathbf{k} Z .
$$

8. Initially straight fibers. Cases in which fibers are initially straight but not parallel are equally simple. From (6.4a) we find that with $\theta_{0}$ constant along each fiber, $\theta$ is also constant along each fiber. Thus, in simple axial tension the fibers remain straight. We can define the fiber angles $\theta_{0}$ and $\theta$ to be zero on some arbitrarily selected fiber; then $\lambda \theta-\theta_{0}$ is zero not only on that fiber but also on all normal lines crossing it, according to $(6.4 \mathrm{~b})$. Then by extension, the same is true everywhere if the cross-section is connected:

$$
\theta=\theta_{0} / \lambda \text {. }
$$

As an example, consider a curvilinear block like that described in Sec. 7 , but with fibers now radial, lying along the lines $\theta_{0}=$ constant. The normal radius of curvature $r_{n}$ is the same as the polar radius $R$. As in any generalized plane deformation, this radius of curvature is conserved in an axial stretching deformation. Thus, the particle initially at $R, \theta_{0}, Z$ moves to $r, \theta, z$, where

$$
r=R, \quad \theta=\theta_{0} / \lambda, \quad z=\lambda Z .
$$

For the general case of fibers that are initially straight but not parallel, each infinitesimal wedge bounded by fibers deforms as the finite wedge in the preceding example did. To describe the deformation analytically, let each fiber be labelled with the fiber angle $\theta_{0}$ on it, and let each normal line be labelled with a value $\xi$ equal to its distance from an arbitrarily chosen normal line $\xi=0$. This distance is unambiguously defined because the normal lines are parallel curves when the fibers are straight. Let $r_{0}\left(\theta_{0}\right)$ be the radius of curvature $r_{n 0}$ of the normal line $\xi=0$. Specification of this function defines the net under consideration. Because the normal lines are parallel, $r_{n 0}$ is then given everywhere by

$$
r_{n 0}=r_{0}\left(\theta_{0}\right)+\xi .
$$

The initial position $\mathbf{X}$ is expressed in terms of the parameters $\xi, \theta_{0}$, and $Z$ by integrating the expression

$$
d \mathbf{X}=\mathbf{a}\left(\theta_{0}\right) d \xi+\mathbf{n}\left(\theta_{0}\right) r_{n 0}\left(\xi, \theta_{0}\right) d \theta_{0}+\mathbf{k}_{0} d Z .
$$


With (2.4) and (8.3), we obtain

$$
\mathbf{X}=\mathbf{a}\left(\theta_{0}\right) \xi+\int_{0}^{\theta_{0}} \mathbf{n}(\alpha) r_{0}(\alpha) d \alpha+\mathbf{k}_{0} Z,
$$

apart from a rigid-body motion.

To obtain a similar expression for the final position $\mathbf{x}$ in terms of the same parameters, we first combine (3.2) to (3.4) as

$$
d \mathbf{x}=\mathbf{a}(\theta) d \xi+\lambda^{-1} \mathbf{n}(\theta) r_{n 0}\left(\xi, \theta_{0}\right) d \theta_{0}+\lambda \mathbf{k} d Z .
$$

Then, by using (2.4), (8.1), and (8.3) here, we obtain

$$
\mathbf{x}=\mathbf{a}\left(\theta_{0} / \lambda\right) \xi+\lambda^{-1} \int_{0}^{\theta_{0}} \mathrm{n}(\alpha / \lambda) r_{0}(\alpha) d \alpha+\lambda \mathbf{k} Z .
$$

9. General Hencky-Prandtl net. For the sake of completeness we consider the unusual cases in which the fibers and normal lines form a Hencky-Prandtl net with fibers neither straight nor parallel. In the undeformed body we label some fiber as $\eta=0$ and some normal line as $\xi=0$. The fiber angle at the point $A$ where they intersect is designated as zero: $\theta_{0 A}=0$. The normal line through any point $B$ on the fiber $\eta=0$ is labelled with a value $\xi$ equal to the fiber angle at $B, \xi=\theta_{0 B}$. Similarly, fibers are labelled with values $\eta$ by taking $\eta$ to be the fiber angle at the point $C$ where the fiber intersects the normal line $\xi=0$. Then at the point $D$ where the normal line $\xi$ and fiber $\eta$ intersect, according to (6.5) the fiber angle is

$$
\theta_{0}=\xi+\eta \text {. }
$$

If we still designate the fiber angle at the particle $A$ as zero after the deformation, then from (6.4) and (9.1), after a little manipulation, we obtain

$$
\theta=\lambda \xi+\eta / \lambda \text {. }
$$

To express the initial position $\mathbf{X}$ in terms of $\xi, \eta$, and $Z$, we integrate the expression

$$
d \mathbf{X}=\mathbf{a}\left(\theta_{0}\right) r_{a 0} d \xi+\mathbf{n}\left(\theta_{0}\right) r_{n 0} d \eta+\mathbf{k}_{0} d Z .
$$

The integrability conditions are found to be

$$
\partial r_{n 0} / \partial \xi=r_{a 0}, \quad \partial r_{a 0} / \partial \eta=-r_{n 0} .
$$

Although it is not feasible to give explicit expressions for the radii of curvature, the relations (9.4) express well-known [14] facts about the radii of a Hencky-Prandtl net.

The final position $\mathbf{x}$ of the same particle is obtained by using (3.2)-(3.4), from which we obtain

$$
d \mathbf{x}=\mathbf{a}(\theta) r_{a 0} d \xi+\lambda^{-1} \mathbf{n}(\theta) r_{n 0} d \eta+\lambda \mathbf{k} d Z .
$$

With (2.4) and (9.2), the integrability conditions again reduce to the conditions (9.4).

10. Stress response. If the structure of the material is symmetric under reflections in planes $Z=$ constant, then in generalized plane deformations there can be no shearing stress on such planes. Consequently, just as in ordinary plane deformations [2], the stress tensor $\boldsymbol{o}$ has the form 


$$
\mathbf{o}=T \mathbf{a a}-P(\mathbf{I}-\mathbf{a a})+S(\mathbf{a n}+\mathbf{n a})+S_{3} \mathbf{k} \mathbf{k} .
$$

Here we use dyadic notation; for example, an is the tensor with Cartesian components $a_{i} n_{i} . \mathrm{I}$ is the unit tensor, with components $\delta_{i i}$.

$T$ is the tension in the fiber direction. Since this stress component can do no work in any kinematically admissible deformation, it represents a reaction to the constraint of inextensibility. Similarly, $P$ is the pressure that appears as the reaction to the constraint of volume incompressibility. The aa component is subtracted away from it for convenience, so that $T$ can represent the total tensile stress in the fiber direction. The reactions $T$ and $P$ cannot be specified by constitutive equations.

$S$ is the shearing stress in the fiber direction, and $S_{3}$ is a normal stress difference:

$$
S=\mathbf{a} \cdot \mathbf{d} \cdot \mathbf{n}, \quad S_{3}=\mathbf{k} \cdot \mathbf{d} \cdot \mathbf{k}-\mathbf{n} \cdot \boldsymbol{d} \cdot \mathbf{n} .
$$

Both of these components are to be specified in terms of the deformation or deformation history by constitutive equations. Since the deformation with respect to the local axes $\mathbf{a}, \mathbf{n}$, and $\mathbf{k}$ is completely specified by the stretch $\lambda$ and the amount of shear $\kappa, S$ and $S_{3}$ are functions of these variables in the case of clastic response, and functionals of the histories of $\lambda$ and $\kappa$ in viscoelastic or plastic response.

For illustration, let us suppose that the material response is clastic, so that $S$ and $S_{3}$ are ordinary functions of $\kappa$ and $\lambda$. The one further assumption that will be needed in order to show that simple axial tension is the same thing as stretching with no shear is that the shearing stress vanishes whenever the shear is zero:

$$
S(0, \lambda)=0 .
$$

Although it is not needed in the present paper, for further applications we mention a relation between $S$ and $S_{3}$ that must hold when the material is elastic. To derive this relation we use the elastic strain energy per unit volume $W$, which depends only on $\kappa$ and $\lambda$ in deformations of the kind considered here. To find expressions for the stresses in terms of $W$, we consider incremental deformations of a material element that was initially a unit cube with fibers parallel to one of its edges. In the deformed state, the faces on which $S_{3}$ acts have areas $1 / \lambda$, and the faces parallel to the fiber direction, on which $S$ acts, have areas $\lambda$. When $\kappa$ and $\lambda$ change, the tensile force acts through a distance $d \lambda$, and the shearing force acts through a distance $d(\kappa / \lambda)$. Other forces acting on the element make cancelling contributions to the work. Hence, on setting the change of stored energy equal to the work done, we obtain

$$
d W=(\lambda S) d(\kappa / \lambda)+\left(S_{3} / \lambda\right) d \lambda .
$$

From this relation we obtain

$$
S=\partial W / \partial \kappa, \quad S_{3}=\lambda \partial W / \partial \lambda+\kappa \partial W / \partial \kappa .
$$

The relation between $S$ and $S_{3}$ is then obtained by eliminating $W$ :

$$
\partial S_{3} / \partial \kappa=S+\lambda \partial S / \partial \lambda+\kappa \partial S / \partial \kappa .
$$

The relations (10.5) can, of course, be derived from the more elaborate three-dimensional form of the constitutive equation, given by Spencer [1] (see also Pipkin [13]).

11. Stress equilibrium. If we assume that material properties are independent of the axial coordinate $Z$, then on using (10.1) in the stress equation of equilibrium, 
$\boldsymbol{\nabla} \cdot \boldsymbol{d}=\mathbf{0}$, we obtain an equation of exactly the same form as for ordinary plane deformations [2]. On separating this into components in the fiber, normal, and axial directions, we obtain

$$
\begin{aligned}
& \mathbf{a} \cdot \nabla T+(P+T) / r_{n}=2 S / r_{a}-\mathbf{n} \cdot \nabla S, \\
& \mathbf{n} \cdot \nabla P-(P+T) / r_{a}=2 S / r_{n}+\mathbf{a} \cdot \nabla S,
\end{aligned}
$$

and $\partial P / \partial z=0$. If the deformation and thus also the shearing stress $S$ are known, these are two first-order equations in characteristic form, determining the variation of $T$ along fibers and the variation of $P$ along normal lines.

In Secs. 5 through 9 we discussed the possibility of states of purely tensile stress in the axial direction. The discussion was in fact entirely kinematical; we considered deformations with $\kappa=0$ everywhere. Under the assumption (10.3), $S$ is zero everywhere in such deformations. Then (11.1) and (11.2) are satisfied by $T=P=0$, and the corresponding surface tractions on the lateral surfaces of the body are zero. Thus, the deformations with $\kappa=0$ everywhere can indeed be supported by a purely axial tension, $\sigma_{z z}=S_{3}(0, \lambda)$.

As an example, let us consider the curvilinear block with fibers along concentric circles, described in Sec. 7, and let us suppose that $\lambda=2 \pi / A$ so that stretching in the axial direction ca uses the block to curl into a tubular shape with its plane faces in contact with one another, as previously described. Suppose now that these faces are bonded together, to form a tube permanently.

When the extending forces are removed after bonding the faces together, the tube retains the same shape but the stress distribution in it is no longer simply a tensile stress in the axial direction. Indeed, since the axial tension has the form

$$
\sigma_{z z}=-P(r, \theta)+S_{3}(0, \lambda),
$$

the condition of no tractions on the ends implies that $\sigma_{z z}=0$ everywhere, and thus that

$$
P(r, \theta)=S_{3}(0, \lambda)\left[H\left(r-r_{1}\right)-H\left(r-r_{2}\right)\right] \text {. }
$$

Here $H$ is the Heaviside unit step function. The step functions take into account the fact that $P$ must be discontinuous at the inner and outer boundaries $r_{1}$ and $r_{2}$, because the conditions of no tractions on these boundaries imply that $P=0$ at $r_{1}-$ and $r_{2}+$.

From (11.2), with $S=0, r_{a}=r$, and $\mathbf{n} \cdot \nabla=-\partial / \partial r$, we obtain

$$
T=-P-r \dot{\partial} P / \partial r \text {. }
$$

Then using (11.4) here, we obtain

$$
T=S_{3}(0, \lambda)\left[r_{2} \delta\left(r-r_{2}\right)-r_{1} \delta\left(r-r_{1}\right)\right]-P .
$$

Thus at interior points, the stress reduces to

$$
\boldsymbol{\delta}=-P(\mathbf{I}-\mathbf{k} \mathbf{k}),
$$

so that the in-plane components amount to an isotropic pressure. From (11.6) we see that this pressure is contained by a finite tensile force in the outermost sheet of fibers equal to $\mathrm{Pr}_{2}$ per unit of length in the axial direction. In the innermost sheet of fibers there is a compressive force of magnitude $P r_{1}$. This suggests that failure by delamination may occur at the inner boundary if the inner fibers are not supported against buckling.

The physical interpretation of stress concentration layers like those in (11.6), taking into account small but finite fiber extensibility, has been discussed elsewhere [11-13]. 
12. Superposed plane deformations. We now briefly verify that the equations governing plane deformations superposed on simple axial tension have exactly the same forms as for ordinary plane deformations (with $\lambda=1$ ), if the state of simple axial tension is used as the reference state. So far as the equilibrium equations are concerned, there is nothing to verify since they do not involve the reference configuration. For the kinematical relations, we label quantities evaluated in the simple tensile state with the subscript unity, so that, for example, the quantities without subscripts in (6.1) to (6.3) have the subscript unity in the new notation. Then by using (6.1) in (3.5) we obtain

$$
\mathbf{a}_{1} \cdot \nabla_{1}=\mathbf{a} \cdot \nabla, \quad \mathbf{n}_{1} \cdot \nabla_{1}=(\mathbf{n}+\kappa \mathbf{a}) \cdot \nabla, \quad \mathbf{k}_{1} \cdot \nabla_{1}=\mathbf{k} \cdot \nabla
$$

Similarly, by using (6.2) or (6.3) in the compatibility conditions (4.4) and (4.6), we obtain

$$
\mathbf{a} \cdot \nabla\left(\theta-\theta_{1}-\kappa\right)=\kappa \mathbf{n} \cdot \nabla \boldsymbol{\theta}, \quad r_{n}=r_{n 1} .
$$

Thus, $\lambda$ is eliminated when the stretched state is used as reference.

It follows that in cases admitting simple axial tension, the solution of any plane problem can be interpreted as the solution of a generalized plane-strain problem, merely by accounting for the parametric dependence of the shearing stress $S$ on the parameter $\lambda$. Of course, the relation of the initial (unstretched) state to the reference (stretched) state depends on the amount of stretch. This relation is given parametrically by (7.6) and (7.8) for all cases involving initially parallel fibers, which include all previously-published solutions.

\section{REFERENCES}

[1] A. J. M. Spencer, Deformations of fibre-reinforced materials, Oxford University Press, London, 1972

[2] A. C. Pipkin and T. G. Rogers, Plane deformations of incompressible fiber-reinforced materials, J. Appl. Mech. 38, 634 (1971)

[3] T. G. Rogers and A. C. Pipkin, Small deflections of fiber-reinforced beams or slabs, J. Appl. Mech. 38, 1047 (1971)

[4] A. C. Pipkin and T. G. Rogers, A mixed boundary value problem for fiber-reinforced materials, Quart. Appl. Math. 29, 151 (1971)

[5] T. G. Rogers and A. C. Pipkin, Finite lateral compression of a fiber-reinforced tube, QJMAM 24, $311(1971)$

[6] G. C. Everstine and T. G. Rogers, $A$ theory of maching of fiber-reinforced materials, J. Comp. Mat. 5, 94 (1971)

[7] B. C. Kao and A. C. Pipkin, Finite buckling of fiber-reinforced columns, Acta Mechanica 13, 265 (1972)

[8] A. H. England, The stress boundary value problem for an ideal fibre-reinforced material, JIMA 9, $310(1972)$.

[9] A. H. England and T. G. Rogers, Plane crack problems for ideal fibre-reinforced materials, QJMAM 26, 303 (1973)

[10] A. C. Pipkin and V. M. Sanchez, Existence of solution of plane traction problems for ideal composites, SIAM J. Appl. Math. 26, 213 (1974)

[11] G. C. Everstine and A. C. Pipkin, Stress channelling in transversely isotropic elastic composites, ZAMP 22, 825 (1971)

[12] G. C. Everstine and A. C. Pipkin, Boundary layers in fiber-reinforced materials, J. Appl. Mech. 40, 518 (1973)

[13] A. C. Pipkin, Finite deformations of ideal fiber-reinforced composites, in Micromechanics, G. P. Sendeckyi, ed., Academic Press, New York, 1973

[14] W. Prager, and P. G. Hodge, Theory of perfectly plastic solids, Dover, New York, 1968 